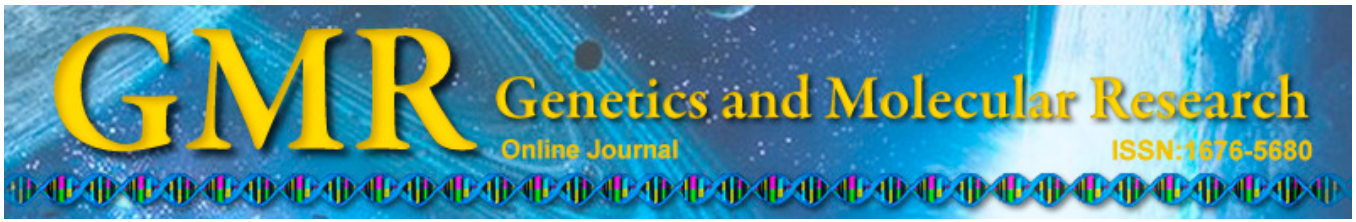

\title{
Association between five common polymorphisms in microRNA genes and the risk of gastric cancer: a meta-analysis
}

\author{
Y.P. Gu ${ }^{1}$, Q.Y. Yuan ${ }^{1}$, H. Zhang' ${ }^{2}$, C.J. Wang ${ }^{2}$ and F. Zhou ${ }^{1}$ \\ ${ }^{1}$ Department of Gastroenterology, Shanghai East Hospital, \\ Tongji University School of Medicine, Shanghai, China \\ ${ }^{2}$ Department of General Surgery, Shanghai East Hospital, \\ Tongji University School of Medicine, Shanghai, China \\ Corresponding author: H. Zhang \\ E-mail: huizhang_easth@163.com
}

Genet. Mol. Res. 14 (3): 8375-8387 (2015)

Received November 3, 2014

Accepted April 6, 2015

Published July 28, 2015

DOI http://dx.doi.org/10.4238/2015.July.28.4

\begin{abstract}
Gastric cancer (GC) is a prevalent disease with a high mortality rate, especially in developing countries. Accumulating evidence suggests that single nucleotide polymorphisms in microRNA (miRNA) genes might influence the susceptibility to GC; such sequence variation might contribute to the development of disease by altering crucial cellular pathways. In this study, we assessed the correlation between the miR-146a G>C, miR-196a-2 C > T, miR-499 T>C, miRNA$27 \mathrm{a} A>\mathrm{G}$, and miRNA-149 $\mathrm{T}>\mathrm{C}$ polymorphisms and the susceptibility to GC. A comprehensive literature search for relevant studies published prior to August 2014 was conducted using PubMed/Medline, Embase, Web of Science, the Cochrane Library, and CNKI databases along with Google Scholar. Meta-analysis was performed using odds ratios (ORs) and 95\% confidence intervals (CIs) as effect measures, incorporating 19 studies encompassing 8285 patients and 10,716 controls. Allelic, dominant, recessive, homozygous, and heterozygous genetic models were examined. Pooled results showed that none of the
\end{abstract}


five polymorphisms studied were statistically related to GC. Stratified analyses by ethnicity and source of controls were conducted for miR146a $\mathrm{G}>\mathrm{C}$ and miR-196a-2 $\mathrm{C}>\mathrm{T}$. Subgroup analysis suggested that the miR-146a G allele might increase the risk of GC in hospital-based case-control (HCC) but not in population-based case-control studies (HCC: recessive model: $\mathrm{OR}=1.23,95 \% \mathrm{CI}=1.10-1.37, \mathrm{P}<0.001$; heterozygous model: $\mathrm{OR}=1.19,95 \% \mathrm{CI}=1.06-1.34, \mathrm{P}=0.004$ ). Overall, this meta-analysis failed to detect an association between five common miR-146a gene polymorphisms and GC susceptibility. However, this does not necessarily completely rule out a correlation between miRNA polymorphisms and GC susceptibility.

Key words: Gastric cancer; MicroRNA; Polymorphism; Meta-analysis

\section{INTRODUCTION}

Gastric cancer (GC) is one of the most common cancers worldwide, and causes the second highest mortality. Although the situation in developed countries is improving steadily, a high incidence rate still exists in Eastern Asia, South America, and Eastern Europe (Jemal et al., 2011). In 2010, it was estimated 287,851 deaths due to GC in China alone (Chen et al., 2014). Several environmental factors have been identified that show correlation with GC, including chronic Helicobacter pylori infection and tobacco consumption. In addition to modification of contributing environmental factors, the early diagnosis of GC has been shown to be important to the outcome of patients with GC as well. In Japan, the mortality rate of patients with GC has declined dramatically through diagnosis of the disease at an early stage via the introduction of photofluorography screening (Lordick et al., 2014).

MicroRNAs (miRNAs, miRs) are endogenous RNAs that can pair with the messenger RNAs of protein-coding genes and direct their post-transcriptional repression. miRNAs play critical roles in the regulation of cellular proliferation, differentiation, and apoptosis. Primary miRNA transcripts are processed to produce pre-miRNAs, which fold into a hairpin structure and are cleaved to produce mature miRNAs from one or both arms (Bartel, 2009). It is estimated that $50 \%$ of human genes might be regulated by miRNAs. Sequence variation in miRNAs could contribute to the development of disease by altering crucial cellular pathways, in particular those involved in carcinogenesis and cancer progression (Wojcicka et al., 2014). Furthermore, the expression of miRNAs is highly tissue and disease-specific.

Single nucleotide polymorphism (SNP) is the variation of a single nucleotide (A, T, $\mathrm{C}$, or $\mathrm{G}$ ) in the genome. SNPs in miRNA regulatory pathways have been widely shown to be correlated with the pathogenesis of cancer. For example, a G/C SNP in pre-miRNA-146a has been demonstrated to be related to the development of thyroid, prostate, and familial breast/ ovarian cancers through its regulation of the level of mature miRNA-146a production (Kogo et al., 2011). Overall, SNPs in miRNAs are hypothesized to be promising biomarkers for cancers of particular organs, such as GC.

In this meta-analysis, several studies were reviewed and five common SNPs in miRNA genes, including miRNA-146a rs2910164, miRNA-196a-2 rs11614913, miRNA-27a rs895819, miRNA-499 rs3746444, and miRNA-149 rs2292832, were analyzed to determine their role in the development of GC. However, some of the studies were inconclusive due to 
the lack of adequate samples. Although several previous meta-analyses have been described that investigated the relevance between SNPs in miRNA genes and GC risk, the contradictions between different studies made it impossible to obtain a decisive estimation of the association. For example, Zeng et al. (2010) and Zhou et al. (2012a) concluded that the $\mathrm{C}$ to $\mathrm{G}$ variation in miRNA-149a was significantly associated $(\mathrm{P}<0.05)$ with $\mathrm{GC}$; whereas Hishida et al. (2011), Ahn et al. (2012), Dikeakos et al. (2014), Kupcinskas et al. (2014) found no significant association $(\mathrm{P}>0.05)$.

In addition, whereas several previous meta-analyses regarding associations between the five common SNPs in miRNA genes and GC have been published, these meta-analyses did not include many eligible studies (Chu et al., 2011; Qiu et al., 2011a,b; Xu et al., 2011; Guo et al., 2012; Zhang et al., 2012a; Wang et al., 2012a,b,c,d,e, 2013a; Wu et al., 2013a). Therefore, we performed an updated and comprehensive meta-analysis on published studies to investigate the correlation between the five common SNPs in miRNA genes and GC susceptibility; and, ideally, to provide evidence useful for the early diagnosis of GC.

\section{MATERIAL AND METHODS}

\section{Search strategy}

PubMed/Medline, Embase, Web of Science, the Cochrane Library, CNKI (Chinese National Knowledge Infrastructure) databases and Google Scholar were searched with the following terms: ("polymorphism" or "SNP" or "gene mutation" or "genetic variants") and ("stomach neoplasms" or "stomach carcinoma" or "stomach cancer" or "gastric cancer") and ("microRNAs" or "miRNAs" or "primary microRNA" or "primary miRNA"). Articles published prior to August 2014 were identified. The reference lists of retrieved articles were manually checked for other relevant publications.

\section{Study selection}

The following criteria had to be satisfied by eligible studies: a) case-control studies covering the association between the five chosen common miRNA gene SNPs and susceptibility to $\mathrm{GC}$; b) sufficient requirements for estimating odds ratios (ORs) and their $95 \%$ confidence interval (CIs) must have been satisfied; and c) the diagnosis of GC was histopathologically confirmed. Studies were excluded according to the following criteria: a) not a case-control study; b) no usable data reported by the study; and c) duplicate of previous publications.

\section{Data extraction}

Data from the retrieved studies were extracted by two reviewers independently (Y.P. $\mathrm{Gu}$ and Q.Y. Yuan). The following information was documented for each study: the name of the first author, publication year, country of origin, ethnicities of subjects, age of subjects, male percentage of subjects, genotyping methods, source of controls, number of subjects, frequencies of alleles and genotypes, and indication of Hardy-Weinberg equilibrium (HWE) in the controls. Different ethnicities were categorized as "Caucasian" or "Asian". In cases of conflicting evaluations, two of the authors discussed the issues to reach a consensus; if no agreements could be reached, a third author would decide. 


\section{Quality assessment}

The Newcastle-Ottawa Quality Assessment Scale (NOS) was applied for methodological quality assessment. This tool is used as a checklist, and contains eight items, categorized into selection, comparability, and exposure (for case-control studies only) groups. A series of options is provided for each item, and a star system is adopted for assessment. For a highquality study, one star is awarded for each item in selection and exposure and two for comparability. The NOS scores of identified studies ranged between zero to nine stars. Studies that received scores of more than six stars were considered to be of high quality and were included in our meta-analysis. The supporting NOS checklist is available in Supplementary file.

\section{Statistical analysis}

The strength of the associations between the five SNPs and the susceptibility to GC were assessed by ORs corresponding to $95 \%$ CIs. Five genetic models (allelic, dominant, recessive, homozygous, and heterozygous) were examined by meta-analysis. A Z-test was applied to assess the statistical significance of the pooled ORs with $\mathrm{P}<0.05$ considered to be statistically significant. For miR-146a $(\mathrm{G}>\mathrm{C})$ and miR-196a-2 $(\mathrm{C}>\mathrm{T})$ variants, subgroup analyses were performed by ethnicity (Asian/Caucasian) and source of controls [hospital-based casecontrol study (HCC) and population-based case-control study (PCC)].

The heterogeneities, i.e., the variation in outcomes between different studies, were tested with Cochran's Q-statistic. A $\mathrm{P}_{\mathrm{h}}>0.05$ illustrated the lack of heterogeneity; subsequently, the pooled OR estimating of the studies without heterogeneity was calculated by the Mantel-Haenszel method using a fixed-effect model. Otherwise, for the random-effect model, the DerSimonian and Laird method was conducted. The $\chi^{2}$ test was applied to test whether the genotype frequencies of the controls were consistent with HWE. The hypothesis that the controls were in HWE was rejected if the P value was less than 0.05 . To assess the reliability of our study, a one-way sensitivity analysis was performed. Individual single studies were omitted sequentially followed by re-analysis to inspect the impact of each study on the overall OR.

A funnel plot was adopted to detect publication bias. The standard error of $\log (\mathrm{OR})$ of each study was plotted against its $\log (\mathrm{OR})$ in the plot. The funnel plot asymmetry was evaluated by the Egger linear regression test on the natural logarithm scale of the OR. All statistical analyses were performed using the STATA Version 12.0 software (Stata Corp., College Station, TX, USA), incorporating two-sided P values.

\section{RESULTS}

\section{Study characteristics}

Sixty-five relevant articles were retrieved based on the search terms. According to the inclusion criteria, 49 articles were excluded. Eventually, 19 case-control studies from 16 articles (Okubo et al., 2010; Peng et al., 2010; Sun et al., 2010; Zeng et al., 2010; Hishida et al., 2011; Ahn et al., 2012; Ma and Zheng, 2012; Zhang et al., 2012b; Zhou et al., 2012a,b; Wang et al., 2013b; Wu et al., 2013b; Yang et al., 2014; Dikeakos et al., 2014; Kupcinskas et al., 2014; $\mathrm{Pu}$ et al., 2014) were included in our meta-analysis, with 8285 patients and 10,716 controls in total. The details of the selection process are presented in Figure 1. Overall, of the 19 eligible 
studies, 17 were conducted on individuals of Asian descent and two were on Caucasians; nine provided data from HCC and ten from PCC studies. In addition, several different genotyping methods were utilized in the retrieved studies. Polymerase chain reaction-restriction fragment length polymorphism (PCR-RFLP) was used by ten studies; real-time PCR (qPCR) was conducted by six studies; PCR with confronting two-pair primers (PCR-CTPP) was adopted by one study; and a single study applied locus-specific PCR (LS-PCR) for genotyping. Genotype distributions in the control groups were consistent with HWE, except for those in three studies (Okubo et al., 2010; Yang et al., 2014; Pu et al., 2014). The NOS scores of the selected studies ranged from six to nine stars, which were moderately high. The details of the methodologies and characteristics of the eligible studies are compiled in Table 1.

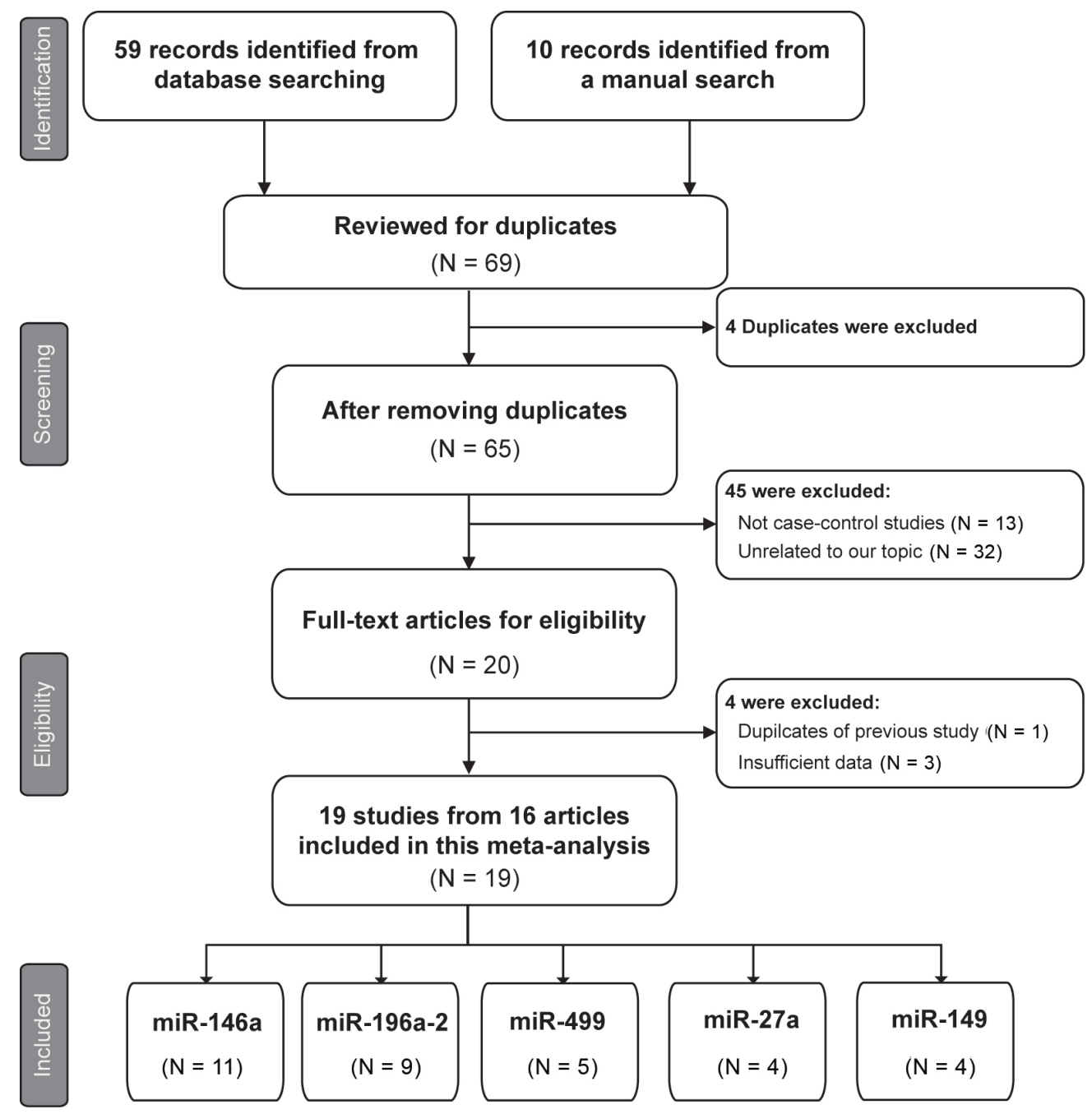

Figure 1. Flow chart of the literature search and study selection. 
Y.P. Gu et al.

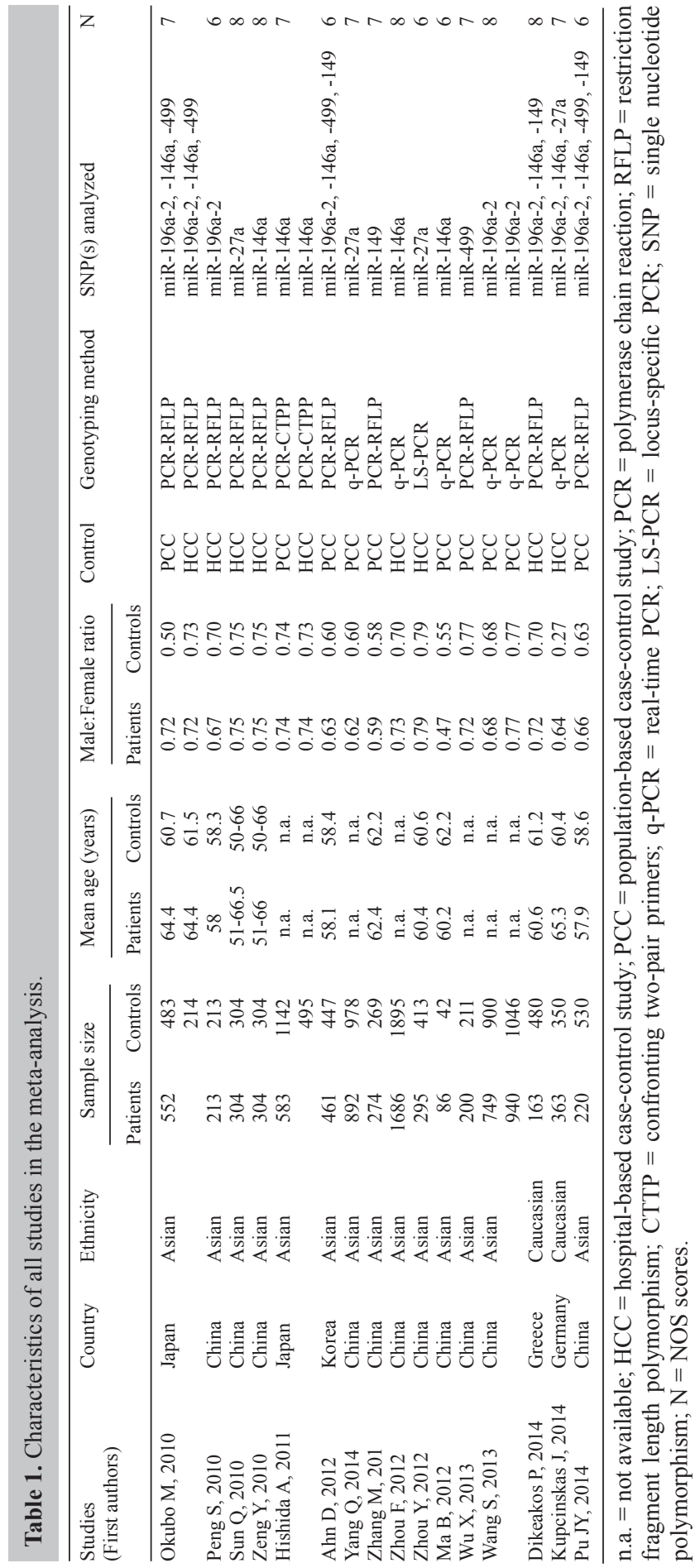




\section{Associations between the five SNPs in the miRNA genes and GC risk}

In total, five SNPs in miRNA-coding genes were assessed in this meta-analysis, including miRNA-146a rs2910164 (G>C), miRNA-196a-2 rs11614913 (C>T), miRNA-27a rs895819 (A>G), miRNA-499 rs3746444 (T>C), and miRNA-149 rs2292832 (T>C). To further inspect the association between miRNA-SNPs and the susceptibility to GC among different ethnicities, the allele and genotype distributions of the miR-146a $(C>G)$ and miR-196a-2 $(\mathrm{T}>\mathrm{C})$ variants were compared between individuals of Asian and Caucasian descent. HCC and PCC subgroups were also analyzed separately to avoid potential bias. A quantitative summary of our results is presented in Table 2 .

\section{MiR-146a (G>C) polymorphism and risk of $\mathrm{GC}$}

The association of the miR-146a $(\mathrm{G}>\mathrm{C})$ polymorphism with $\mathrm{GC}$ was investigated in 11 studies. Of these, nine were performed using Asian populations, whereas only two were based on Caucasians. Both fixed- and random-effect models were applied to pool the results, as heterogeneity $\left(\mathrm{P}_{\mathrm{h}}>0.05\right)$ was not confirmed in every study. For the miR-146a $(\mathrm{G}>\mathrm{C})$ polymorphism, no statistically significant association with $\mathrm{GC}$ was observed under the allelic (OR $=1.04,95 \% \mathrm{CI}=0.93-1.16, \mathrm{P}=0.496)$, dominant $(\mathrm{OR}=1.02,95 \% \mathrm{CI}=0.88-1.19, \mathrm{P}=0.772)$, or homozygous $(\mathrm{OR}=1.08,95 \% \mathrm{CI}=0.87-1.34, \mathrm{P}=0.473)$ models, nor could a clear correlation be identified in the study when stratified by ethnicity (Figure 2A).

However, the subgroup analysis of HCC studies suggested that the miR-146a polymorphism $\mathrm{G}$ allele might increase the susceptibility to $\mathrm{GC}$ (recessive model: $\mathrm{OR}=1.23,95 \% \mathrm{CI}=$ 1.10-1.37, $\mathrm{P}<0.001$; heterozygous model: $\mathrm{OR}=1.19,95 \% \mathrm{CI}=1.06-1.34, \mathrm{P}=0.004$ ) (Figure 2B). In contrast, no changes in cancer susceptibility were correlated with the miR-146a $(\mathrm{G}>\mathrm{C})$ polymorphism in the $\mathrm{PCC}$ studies (allelic model: $\mathrm{OR}=0.96,95 \% \mathrm{CI}=0.83-1.12, \mathrm{P}=0.611$; dominant model: $\mathrm{OR}=0.93,95 \% \mathrm{CI}=0.82-1.05, \mathrm{P}=0.231$; recessive model: $\mathrm{OR}=0.94$, $95 \% \mathrm{CI}=0.79-1.11, \mathrm{P}=0.168$; homozygous model: $\mathrm{OR}=0.90,95 \% \mathrm{CI}=0.75-1.08, \mathrm{P}=0.251$; heterozygous model: $\mathrm{OR}=0.96,95 \% \mathrm{CI}=0.80-1.15, \mathrm{P}=0.686$ ) (Figure $2 \mathrm{~B}$ ).

\section{MiR-196a-2 (C>T) polymorphism and the risk of GC}

In the meta-analysis, nine studies addressed the association of the miR-196a-2 $(\mathrm{C}>\mathrm{T})$ polymorphism with GC risk, among which seven were based on Asians and two on Caucasians. A random-effect model was applied to the studies with significant heterogeneity $\left(\mathrm{P}_{\mathrm{h}}<\right.$ 0.05), whereas a fixed-effect model was conducted under the dominant model in the Asian and PCC subgroups $\left(\mathrm{P}_{\mathrm{h}}>0.05\right)$. For the miR-196a-2 $(\mathrm{C}>\mathrm{T})$ polymorphism, all five genetic models failed to demonstrate an association with $\mathrm{GC}$ (allelic model: $\mathrm{OR}=1.21,95 \% \mathrm{CI}=0.92-1.58$, $\mathrm{P}=0.167$; dominant model: $\mathrm{OR}=1.23,95 \% \mathrm{CI}=0.95-1.59, \mathrm{P}=0.125$; recessive model: $\mathrm{OR}$ $=1.29,95 \% \mathrm{CI}=0.83-2.00, \mathrm{P}=0.266$; homozygous model: $\mathrm{OR}=1.42,95 \% \mathrm{CI}=0.88-2.28$, $\mathrm{P}=0.152$; heterozygous model: $\mathrm{OR}=1.22,95 \% \mathrm{CI}=0.83-1.80, \mathrm{P}=0.321)$ (Table 2). No association with GC was observed upon stratification by racial descent (Table 2). Finally, the meta-analysis results following further stratification by source of controls also identified no correlation between the miR-196a-2 $(\mathrm{C}>\mathrm{T})$ polymorphism and the susceptibility to $\mathrm{GC}$ in either HCC or PCC subgroups, consistent with the outcomes for the study group as a whole. 
Y.P. Gu et al.

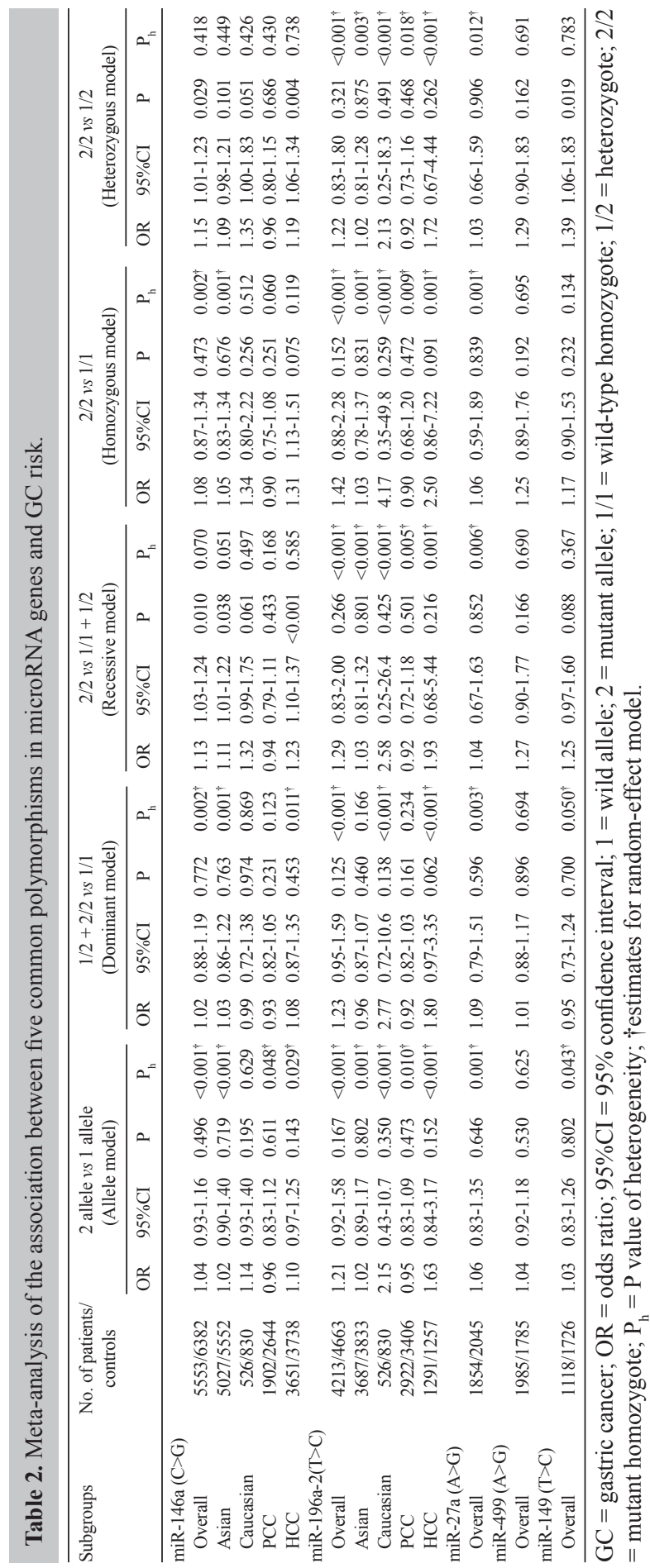


(A)

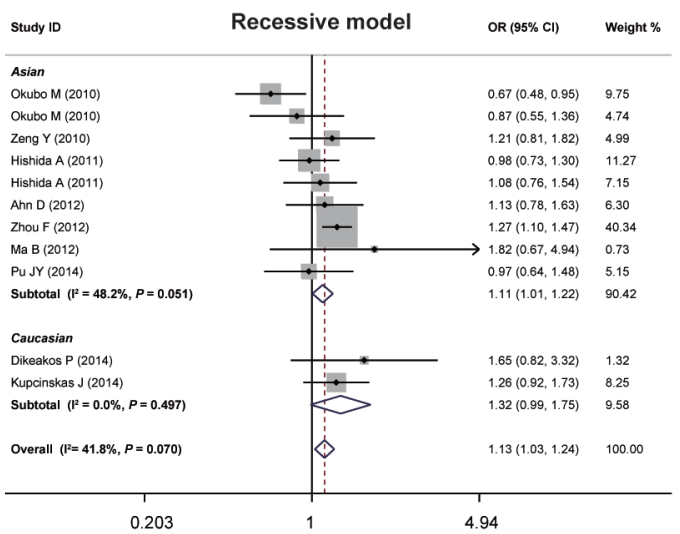

(C)

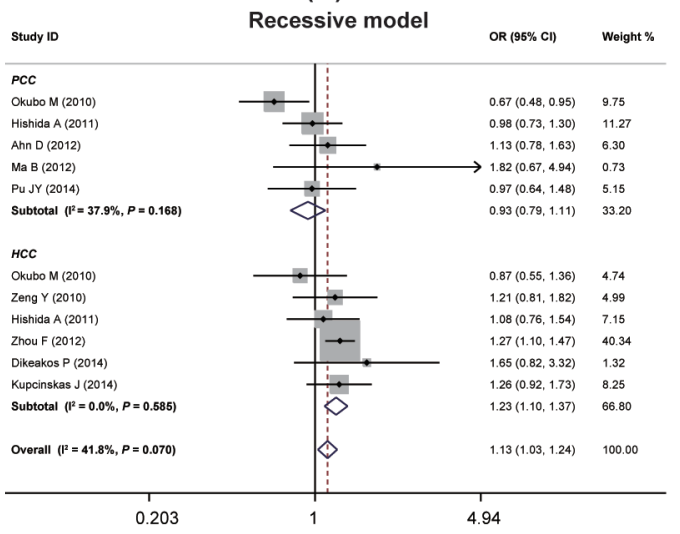

(B)

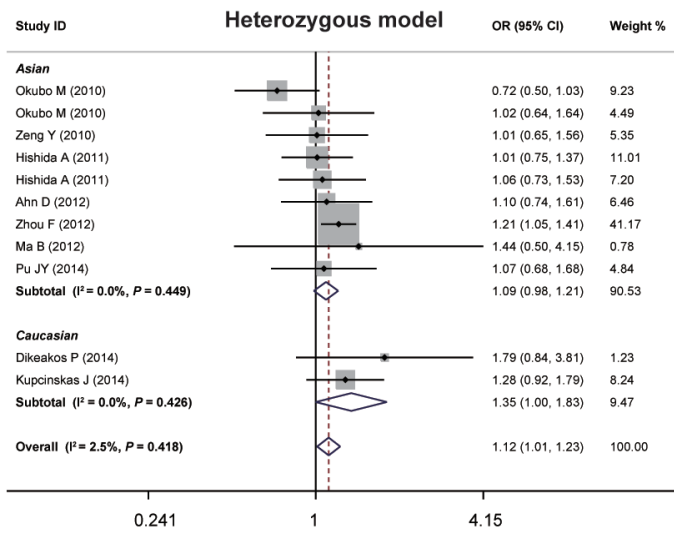

(D)

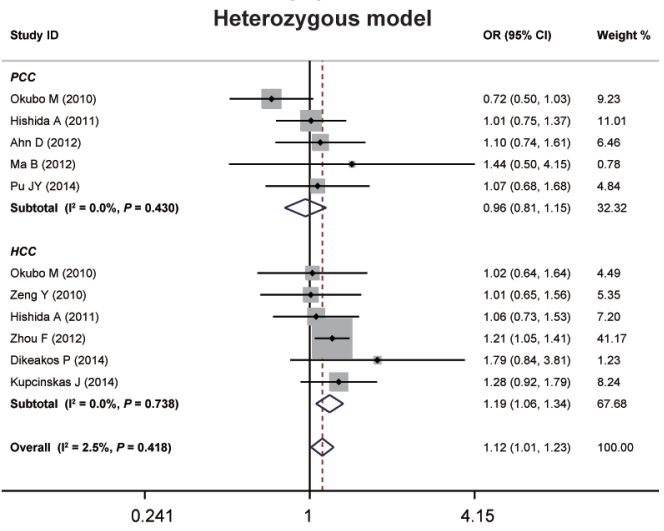

Figure 2. Forest plots of ORs with $95 \%$ CIs for the miR-146a $C>G$ polymorphism and gastric cancer susceptibility. (A) Recessive model for subgroup analysis based on ethnicity; (B) heterozygous model for subgroup analysis based on ethnicity; (C) recessive model for subgroup analysis based on source of control; (D) heterozygous model for subgroup analysis based on source of control.

\section{MiR-27a $(A>G)$, miR-499 $(A>G)$, and miR-149 $(T>C)$ polymorphisms and the risk of GC}

The association between $\mathrm{GC}$ and miR-27a $(\mathrm{A}>\mathrm{G})$ variation was examined by five studies included in the current meta-analysis, and four studies each analyzed miR-499 $(A>G)$ and miR-149 $(\mathrm{T}>\mathrm{C})$ variation and GC risk. Stratification was not performed owing to the small sample sizes for each miRNA-SNP. A random-effect model was applied to miR-27a $(A>G)$ polymorphism analysis for obvious heterogeneity $\left(\mathrm{P}_{\mathrm{h}}<0.05\right)$, whereas a fixed-effect model was utilized for the analysis of miR-499 $(A>G)$ polymorphism, which demonstrated relatively low heterogeneity $\left(\mathrm{P}_{\mathrm{h}}>0.05\right)$. We did not observe a significant association between any of the three miR-SNPs and the risk of GC $(\mathrm{P}>0.05)$. 


\section{Sensitivity analysis and publication bias}

The influence of each study was evaluated by sensitivity analysis on the pooled OR through sequential omission of studies. The results suggested that the overall ORs for the five miRNA gene SNPs were not significantly altered by any of the individual studies. This analysis demonstrated that the eligible studies were statistically robust.

Publication biases of the articles were assessed using Begg's funnel plot and the Egger linear regression test on the metadata. The distributions of the different studies on the funnel plot for each miR-SNP appeared to be symmetrical. In addition, no statistically significant asymmetry was identified by the Egger test (miR-146a: $t=0.40, \mathrm{P}=0.701 ;$ miR-196a-2: $t=$ 1.36, $\mathrm{P}=0.105$; miR-499: $t=-0.60, \mathrm{P}=0.592$; miR-27a: $t=-1.18, \mathrm{P}=0.360$; miR-149: $t=$ $2.59, \mathrm{P}=0.122$ ) (Figure 3). Overall, these analyses detected no evidence of publication bias for the correlation between the five miRNA-SNPs and GC susceptibility.

\section{(A) miR-146a}

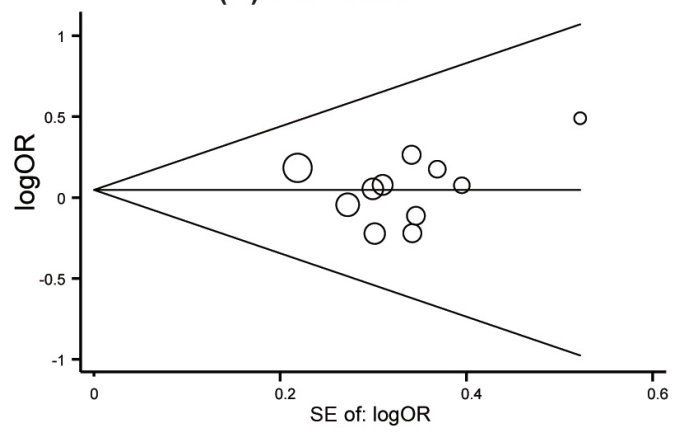

(B) miR-196a-2

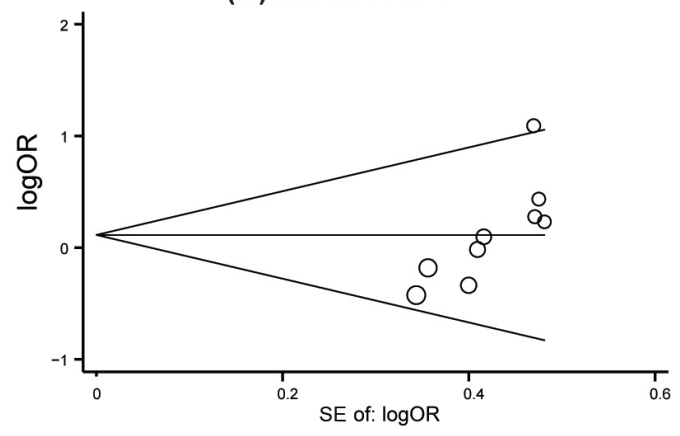

Figure 3. Funnel plots of studies included in the meta-analyses. (A) miR-146a; (B) miR-196a-2.

\section{DISCUSSION}

In the current study, we performed a meta-analysis on miR-146a G>C (rs2910164), miR-196a-2 C $>$ T (rs11614913), miR-499 T>C (rs3746444), miRNA-27a A $>$ G (rs895819), and miRNA-149 T>C (rs2292832) gene polymorphisms and GC risk in 16 case-control studies, encompassing 19,001 subjects (8285 patients and 10,716 controls). Since miRNAs have been demonstrated to play a crucial role in gastric carcinogenesis, we expected that these polymorphisms might modify the risk of GC. However, our meta-analysis results do not support this hypothesis.

According to Wu et al. (2014), miR-146a density was correlated with GC. Specifically, miR-146a density was positively associated with apoptosis rates in $H$. pylori-positive gastric tissues, which might provide a link to the gastric carcinogenesis process. Conversely, a negative effect of miR-146a on lymph node metastasis was shown to be closely related to tumor development. Therefore, we included the rs 2910164 polymorphism in pre-miR-146a in our study to assess its association with GC. However, we identified no statistically significant associations between SNP rs2910164 and the susceptibility to GC in either Caucasians or Asians. This result is consistent with previous studies (Dikeakos et al., 2014; Kupcinskas et al., 2014; Pu et al., 2014). Our results did suggest, on the other hand, that the G allele was correlated with an increased GC risk in HCC-but not in PCC-based studies. The inconsistency 
in our results suggests a potential influence of the $\mathrm{C}$ allele on susceptibility to other diseases, as all the controls in the studies exhibiting association with GC risk originated from hospital populations. Furthermore, considering the limited sample size in the stratified HCC studies, the validity of this result might not be high.

Annexin A1 (ANXA1), a potential tumor suppressor, has been reported to be a direct target of miR-196a (Luthra et al., 2008). In addition, high expression levels of miR-196a have been shown to promote cancer cell migration and invasion through the regulation of homeobox genes (Kim et al., 2009). Therefore, SNPs in miR-196a are suspected to be associated with the susceptibility to human cancer. However, according to our meta-analysis, no association could be identified between the miR-196a-2 C $>$ T polymorphism and GC risk, and we observed no difference between groups upon stratification by ethnicity or source of control. These results contradict the findings of Wang et al. (2013b), Dikeakos et al. (2014), Kupcinskas et al. (2014); this inconsistency might be due to the larger sample size of the current study. On the other hand, our results do not necessarily obviate the possibility that other polymorphic variants in miR-196a might be associated with GC susceptibility.

Significant upregulation of miR-27a has been observed in GC tissues and high expression levels of miR-27a have been correlated with poor tumor histological grade (Qiu et al., 2011b). These findings indicate the important role of miR-27a in gastric carcinogenesis. miR-499 is specifically expressed in skeletal muscle and cardiac cells (van Rooij et al., 2009). miR-149 has been shown to be able to suppress breast cancer cell migration, invasion and metastasis by targeting GIT1, suggesting a potential role of miR-149 variation in the development of human cancer (Chan et al., 2014). However, three of our studied SNPs, including those in miR-499 (rs3746444), miRNA-27a (rs895819), and miRNA-149 (rs2292832) did not exhibit a correlation with GC. This discrepancy with previous research might be attributed to a lack of stratified analysis and the small sample size available for the studied SNPs. Nonetheless, the possible correlation of other SNPs with GC cannot be ruled out.

The principal results of the present study suggest that miR-146a, miR-196a-2, miR-499, miRNA-27a, and miRNA-149 gene polymorphisms might not be associated with the risk of GC. In particular, the conflicting result from HCC subgroup stratification for miR-146a indicates the limitations of previous studies related to the source of controls. Nevertheless, the maximum number of eligible case-control studies were covered in our meta-analysis, including those most recently published (Dikeakos et al., 2014; Kupcinskas et al., 2014; Pu et al., 2014). However, certain issues remain to be addressed in interpreting our meta-analysis results. First, the majority of the subjects included in our study were of Asian descent $(17,645 / 19,001)$. This factor limits the general application of the results. Further studies with more Caucasian and African subjects are recommended. Second, although our results were statistically sufficient, the overall sample sizes in our study and in the stratified subgroups were still relatively small. Additionally, the efficacy of the various genotyping methods utilized in the different eligible studies might have had an impact on the results; further study is required to minimize the potential effects of such aspects. Furthermore, individual genetic factors, and the biological characteristics of tumors and their interaction with environment might exert an influence on cancer susceptibility and carcinogenesis. For example, chronic Helicobacter pylori infection is believed to be associated with GC carcinogenesis; improvement in sanitation and diet is an important component of the decreasing incidence rate in developed countries (Karimi et al., 2014).

In summary, this meta-analysis failed to detect an association between miR-146a $\mathrm{G}>\mathrm{C}$ (rs2910164), miR-196a-2 C > T (rs11614913), miR-499 T>C (rs3746444), miRNA-27a A > G 
(rs895819), and miRNA-149 T>C (rs2292832) gene polymorphisms and the risk of GC. However, this finding does not necessarily completely rule out the potential for microRNA polymorphisms to serve as GC biomarkers. Future studies on this topic are therefore recommended.

\section{Conflicts of interest}

The authors declare no conflict of interest.

\section{Supplementary material}

\section{REFERENCES}

Ahn DH, Rah H, Choi YK, Jeon YJ, et al. (2012). Association of the miR-146aC $>$ G, miR-149T $>$ C, miR-196a2T $>$ C, and miR-499A $>$ G polymorphisms with gastric cancer risk and survival in the Korean population. Mol. Carcinog. 52 (Suppl 1): E39-E51.

Bartel DP (2009). MicroRNAs: target recognition and regulatory functions. Cell 136: 215-233.

Chan SH, Huang WC, Chang JW, Chang KJ, et al. (2014). MicroRNA-149 targets GIT1 to suppress integrin signaling and breast cancer metastasis. Oncogene 33: 4496-4507.

Chen W, Zheng R, Zhang S, Zhao P, et al. (2014). Annual report on status of cancer in China, 2010. Chin. J. Cancer Res. 26: 48-58.

Chu H, Wang M, Shi D, Ma L, et al. (2011). Hsa-miR-196a2 Rs11614913 polymorphism contributes to cancer susceptibility: evidence from 15 case-control studies. PLoS One 6: e18108.

Dikeakos P, Theodoropoulos G, Rizos S, Tzanakis N, et al. (2014). Association of the miR-146aC $>$ G, miR-149T $>$ C, and miR-196a2T $>$ C polymorphisms with gastric cancer risk and survival in the Greek population. Mol. Biol. Rep. 41: 1075-1080.

Guo J, Jin M, Zhang M and Chen K (2012). A genetic variant in miR-196a2 increased digestive system cancer risks: a meta-analysis of 15 case-control studies. PLoS One 7: e30585.

Hishida A, Matsuo K, Goto Y, Naito M, et al. (2011). Combined effect of miR-146a rs2910164 G/C polymorphism and Toll-like receptor $4+3725 \mathrm{G} / \mathrm{C}$ polymorphism on the risk of severe gastric atrophy in Japanese. Dig. Dis. Sci. 56: 1131-1137.

Jemal A, Bray F, Center MM, Ferlay J, et al. (2011). Global cancer statistics. CA Cancer J. Clin. 61: 69-90.

Karimi P, Islami F, Anandasabapathy S, Freedman ND, et al. (2014). Gastric cancer: descriptive epidemiology, risk factors, screening, and prevention. Cancer Epidemiol. Biomarkers Prev. 23: 700-713.

Kim YJ, Bae SW, Yu SS, Bae YC, et al. (2009). miR-196a regulates proliferation and osteogenic differentiation in mesenchymal stem cells derived from human adipose tissue. J. Bone Miner. Res. 24: 816-825.

Kogo R, Mimori K, Tanaka F, Komune S, et al. (2011). Clinical significance of miR-146a in gastric cancer cases. Clin. Cancer Res. 17: 4277-4284.

Kupcinskas J, Wex T, Link A, Leja M, et al. (2014). Gene polymorphisms of micrornas in Helicobacter pylori-induced high risk atrophic gastritis and gastric cancer. PLoS One 9: e87467.

Lordick F, Allum W, Carneiro F, Mitry E, et al. (2014). Unmet needs and challenges in gastric cancer: the way forward. Cancer Treat. Rev. 40: 692-700.

Luthra R, Singh RR, Luthra MG, Li YX, et al. (2008). MicroRNA-196a targets annexin A1: a microRNA-mediated mechanism of annexin A1 downregulation in cancers. Oncogene 27: 6667-6678.

Ma B and Zheng JZ (2012). Association between microRNA-146a polymorphism and gastric cancer. Chin. J. Gerontol. 32: $3150-3152$

Okubo M, Tahara T, Shibata T, Yamashita H, et al. (2010). Association between common genetic variants in premicroRNAs and gastric cancer risk in Japanese population. Helicobacter 15: 524-531.

Peng S, Kuang Z, Sheng C, Zhang Y, et al. (2010). Association of microRNA-196a-2 gene polymorphism with gastric cancer risk in a Chinese population. Dig. Dis. Sci. 55: 2288-2293.

$\mathrm{Pu}$ JY, Dong W, Zhang L, Liang WB, et al. (2014). No association between single nucleotide polymorphisms in premiRNAs and the risk of gastric cancer in Chinese population. Iran J. Basic Med. Sci. 17: 128-133.

Qiu LX, He J, Wang MY, Zhang RX, et al. (2011a). The association between common genetic variant of microRNA-146a and cancer susceptibility. Cytokine 56: 695-698.

Qiu LX, Wang Y, Xia ZG, Xi B, et al. (2011b). miR-196a2 C allele is a low-penetrant risk factor for cancer development. 
Cytokine 56: 589-592.

Sun Q, Gu H, Zeng Y, Xia Y, et al. (2010). Hsa-mir-27a genetic variant contributes to gastric cancer susceptibility through affecting miR-27a and target gene expression. Cancer Sci. 101: 2241-2247.

van Rooij E, Quiat D, Johnson BA, Sutherland LB, et al. (2009). A family of microRNAs encoded by myosin genes governs myosin expression and muscle performance. Dev. Cell 17: 662-673.

Wang F, Ma YL, Zhang P, Yang JJ, et al. (2012a). A genetic variant in microRNA-196a2 is associated with increased cancer risk: a meta-analysis. Mol. Biol. Rep. 39: 269-275.

Wang F, Sun G, Zou Y, Fan L, et al. (2012b). Lack of association of miR-146a rs2910164 polymorphism with gastrointestinal cancers: evidence from 10206 subjects. PLoS One 7: e39623.

Wang F, Sun GP, Zou YF, Fan LL, et al. (2013a). Quantitative assessment of the association between miR-196a2 rs11614913 polymorphism and gastrointestinal cancer risk. Mol. Biol. Rep. 40: 109-116.

Wang J, Bi J, Liu X, Li K, et al. (2012c). Has-miR-146a polymorphism (rs2910164) and cancer risk: a meta-analysis of 19 case-control studies. Mol. Biol. Rep. 39: 4571-4579.

Wang J, Wang Q, Liu H, Shao N, et al. (2012d). The association of miR-146a rs2910164 and miR-196a2 rs11614913 polymorphisms with cancer risk: a meta-analysis of 32 studies. Mutagenesis 27: 779-788.

Wang P, Xie S, Cui A, Zhang Y, et al. (2012e). miR-196a2 polymorphisms and susceptibility to cancer: A meta-analysis involving 24,697 subjects. Exp. Ther. Med. 3: 324-330.

Wang S, Tao G, Wu D, Zhu H, et al. (2013b). A functional polymorphism in MIR196A2 is associated with risk and prognosis of gastric cancer. Mol. Carcinog. 52 (Suppl 1): E87-E95.

Wojcicka A, de la Chapelle A and Jazdzewski K (2014). MicroRNA-related sequence variations in human cancers. Hum. Genet. 133: 463-469.

Wu D, Wang F, Dai WQ, He L, et al. (2013a). The miR-146a rs2910164 G > C polymorphism and susceptibility to digestive cancer in Chinese. Asian Pac. J. Cancer Prev. 14: 399-403.

Wu K, Yang L, Li C, Zhu CH, et al. (2014). MicroRNA-146a enhances Helicobacter pylori induced cell apoptosis in human gastric cancer epithelial cells. Asian Pac. J. Cancer Prev. 15: 5583-5586.

Wu XJ, Mi YY, Yang H, Hu AK, et al. (2013b). Association of the hsa-mir-499 (rs3746444) polymorphisms with gastric cancer risk in the Chinese population. Onkologie 36: 573-576.

Xu W, Xu J, Liu S, Chen B, et al. (2011). Effects of common polymorphisms rs11614913 in miR-196a2 and rs2910164 in miR-146a on cancer susceptibility: a meta-analysis. PLoS One 6: e20471.

Yang Q, Jie Z, Ye S, Li Z, et al. (2014). Genetic variations in miR-27a gene decrease mature miR-27a level and reduce gastric cancer susceptibility. Oncogene 33: 193-202.

Zeng Y, Sun QM, Liu NN, Dong GH, et al. (2010). Correlation between pre-miR-146a C/G polymorphism and gastric cancer risk in Chinese population. World J. Gastroenterol. 16: 3578-3583.

Zhang H, Su YL, Yu H and Qian BY (2012a). Meta-Analysis of the Association between Mir-196a-2 Polymorphism and Cancer Susceptibility. Cancer Biol. Med. 9: 63-72.

Zhang MW, Jin MJ, Yu YX, Zhang SC, et al. (2012b). Associations of lifestyle-related factors, hsa-miR-149 and hsamiR-605 gene polymorphisms with gastrointestinal cancer risk. Mol. Carcinog. 51 (Suppl 1): E21-E31.

Zhou F, Zhu H, Luo D, Wang M, et al. (2012a). A functional polymorphism in Pre-miR-146a is associated with susceptibility to gastric cancer in a Chinese population. DNA Cell Biol. 31: 1290-1295.

Zhou Y, Du WD, Chen G, Ruan J, et al. (2012b). Association analysis of genetic variants in microRNA networks and gastric cancer risk in a Chinese Han population. J. Cancer Res. Clin. Oncol. 138: 939-945. 